\title{
"Neoliberalismus". Diskussion eines Grundbegriffs zur Analyse sozioökonomischer Gegenwart und zur Reflexion von Bildungsinhalten
}

\author{
"michael.lehner@uni-due.de, Institut für Sachunterricht, Universität Duisburg-Essen \\ **inga.gryl@uni-due.de, Institut für Geographie \& Institut für Sachunterricht, Universität Duisburg-Essen \\ eingereicht am: 01.06.2019, akzeptiert am: 25.07.2019
}

In diesem Artikel wird dem Begriff und dem Phänomen Neoliberalismus überblicksartig nachgegangen. Ziel dieser Diskussion ist es, einen Begriff des Neoliberalen herzuleiten, der sich auch zur Analyse von Bildungsinhalten eignet, um entsprechende Tendenzen herausarbeiten zu können.

Keywords: Neoliberalismus; Mündigkeit, sozioökonomische Bildung, entrepreneurial child

\section{"Neoliberalism". Discussion of a fundamental concept to analyse the socio-economic present a reflect on curricula}

This article explores the term and the phenomenon of neoliberalism. The discussion should supply an analytical potential of the term neoliberalism, which supports the analysis of educational content concerning neoliberal tendencies.

Keywords: Neoliberalism, maturity, socio-economic education, entrepreneurial child

\section{$1 \quad$ Einleitung}

"Kant sah die Fremdbestimmung seiner Mitmenschen in erster Linie in einem ideologischen Missbrauch von Religion durch die Herrschenden bedingt. In den "aufgeklärten" Gesellschaften des frühen 21. Jahrhunderts hingegen ist es vor allem ein um marktliberale Dogmen kreisendes, ökonomi(sti) sches Denken, das die Menschen indoktriniert und einem selbstbestimmten Vernunftgebrauch im Wege steht"(Haarmann, 2019, S. 163).

Die Bedingungen von Unterricht müssen immer in einen „Strukturzusammenhang" (Kron, Jürgens \& Standop 2014: 25) reflektiert werden, da Bildung und damit Unterricht in einer Wechselbeziehung zu gesellschaftlichen Verhältnissen steht. Angetrieben von dieser Grundüberlegung wollten wir Unterrichtsmaterialien auf neoliberale Tendenzen - die „Ideologie der Gegenwart"(Haarmann 2019: 159), wie der eingangs zitierte Aufsatz ein entsprechendes ökonomi(sti)sches Denken auch bezeichnet - untersuchen. Im Zuge die- ses Vorhabens ist uns aufgefallen, dass zum Themenkomplex des Neoliberalismus zwar ein enormer Umfang an Literatur besteht, aber in der fachdidaktischen Debatte, auch nicht im Bereich der GW-Didaktik, kein Überblicksartikel auffindbar ist, der den Begriff Neoliberalismus als Grundlage einer systematischen Analyse vorbereiten würde. Diese Lücke versuchen wir mit diesem Aufsatz zu schließen. Dabei möchten wir nicht nur Definitionen von „Neoliberalismus“ zusammenstellen, sondern auch bereits andenken, wie dieser etwa in Unterrichtsmaterialien erkannt werden kann.

Um entsprechende ökonomi(sti)sche Ansätze in den $\mathrm{zu}$ untersuchenden Unterrichtsmaterialien zu identifizieren, würde sich etwa in Anlehnung an Kuhn (1969) eine paradigmatische Einordnung anbieten. Hierbei ließe sich etwa fragen, welcher ökonomischen Denktradition (z. B. Neoklassik, Keynesianismus, Politische Ökonomie, etc.) die über die Unterrichtsmaterialien transportierte Sichtweise entspricht (vgl. Heine \& Herr 2012). Dieser Ansatz eignet sich insbesondere für Unterrichtsmaterialien, die explizit öko- 
nomische Themen behandeln. Die uns vorliegenden Unterrichtsmaterialien aus dem Bereich des Sachunterrichts weisen jedoch weitgehend einen anderen Charakter auf und sie behandeln über weite Strecken weniger explizit ökonomische Themen. Wenn beispielsweise thematisiert wird, worauf bei gesunder Ernährung zu achten ist (vgl. Jo-Jo 1: 37), oder wie mit Müll umgegangen werden kann (vgl. Schlag nach 1/2: 104-105), lässt sich dieses Material kaum hinsichtlich expliziter ökonomischer Paradigmen einordnen. Wir sind jedoch der Auffassung, dass es dennoch neoliberale Tendenzen aufweisen kann, da wir „Neoliberalismus" als ein ideologisches Projekt erachten, welches über eine ökonomi(sti)sche Sichtweise hinausgeht. In Unterrichtsmaterialien für den Geographieunterricht dürfte sich angesichts der ebenfalls interdisziplinären Ausrichtung des Fachs mit Querschnittsthemen wie Bevölkerungsgeographie und Nachhaltigkeit diese Tendenz teilweise fortsetzen.

Um unsere These, dass neoliberale Tendenzen auch in nicht explizit ökonomischen Bildungsinhalten zu finden sind, zu untersuchen, möchten wir in diesem Artikel der Frage nachgehen, was unter „Neoliberalismus" verstanden werden kann und wie dieser in Erscheinung tritt. Charaktermerkmale, die sich im Zuge dieser Diskussion ableiten lassen, werden wir im letzten Abschnitt (Abschnitt 6) hinsichtlich der aufgeworfenen These reflektieren, um davon Ansätze für eine Analyse von Unterrichtsmaterialien und -praktiken hinsichtlich neoliberaler Tendenzen herzuleiten.

Wirft man nun die Frage nach dem Begriff und dem Phänomen Neoliberalismus auf, so ergeben sich zahlreiche Unterfragen und spannende Nebenschauplätze. Um in diesem Dickicht nicht verlorenzugehen, versuchen wir anhand von vier Perspektiven Konturen des Neoliberalismus abzuleiten. Die erste Frage, die wir uns diesbezüglich stellen, zielt auf unterschiedliche Bedeutungszuschreibungen des Begriffs. So wird sich zeigen, dass "Neoliberalismus“, etwa in unterschiedlichen Kontexten, durchaus unterschiedliche Bedeutungen transportiert. Darüber hinaus steht "Neoliberalismus" auch in engen, sich teilweise überschneidenden Zusammenhängen mit anderen Begriffen, wie beispielsweise "Neoklassik“" (Abschnitt 2). Ausgehend von Problemen, die sich aus dieser Diskussion auf begrifflicher Ebene ergeben, betrachten wir aus ideengeschichtlicher Perspektive die Frage nach Gemeinsamkeiten und Unterschieden zwischen Klassik, Neoklassik, Liberalismus und Neoliberalismus (Abschnitt 3). Die Zusammenhänge, die sich aus dieser Perspektive abzeichnen und wir als "neoliberales Projekt" bezeichnen werden, untersuchen wir aus strukturgeschichtlicher Perspektive exemplarisch hinsichtlich dessen Auswirkung auf die europäische Integrationsweise (Abschnitt 4). Die vierte Perspektive, die wir einnehmen, bezieht sich auf die Subjektebene. Diesbezüglich fragen wir nach veränderten Regierungspraktiken in einer neoliberal geprägten Gegenwart (Abschnitt 5).

\section{2 „Neoliberalismus“ als analytischer Be- griff - ein Problemaufriss}

Mit folgendem Abschnitt sollen die Bedeutungsvielfalt des Begriffs „Neoliberalismus“ systematisiert werden und wer könnte sich als archetypischer Ausgangspunkt besser eignen als Christian Lindner (Bundesvorsitzender der FDP), um eine solche Diskussion zu eröffnen:

„Ich kämpfe nicht gegen Windmühlen.“, antwortet Lindner auf die Frage, ob er jemals darüber nachgedacht hätte, das Wort "neoliberal“ mit positiver Bedeutung wiederzubeleben (Nienhaus 2017). Aus Lindners Aussage lassen sich immerhin zwei Hinweise auf den Gebrauch des Attributs neoliberal ableiten. Neoliberal lässt sich als politischer Kampfbegriff erkennen und verweist auf eine polemische Dimension, die eine Politik, welche als neoliberal identifiziert wird, eines „Raubtierkapitalismus" bezichtigt. Außerdem wird deutlich, dass Lindner neoliberal nicht als treffende Selbstzuschreibung verwendet, da er die negative Rahmung des Begriffs hinnehmend anerkennt. Jenseits der Polemik, welche dieser Begriff ermöglicht, wird "Neoliberalismus" allerdings auch analytisch von einer großen Gruppe Sozialwissenschaftler/innen verwendet, um einen globalen Siegeszug in der Form bestimmter sozioökonomischer Transformationsprozesse seit Mitte der 1970er Jahre zu identifizieren (Birch \& Mykhnenko 2010; Crouch 2011; Harvey 2012). Diese beiden sehr unterschiedlichen Zugänge zu dem Begriff lassen „Neoliberalismus“ in einem interessanten Spannungsfeld verorten, was ein merkwürdiges Phänomen erscheinen lässt: ein herrschender Neoliberalismus - ohne Neoliberale (zumindest ohne jene, die sich selbst als solche identifizieren).

Neben dieser widersprüchlichen Zuspitzung lassen sich in unterschiedlichen Regionen unterschiedliche begriffliche Konstellationen und Bedeutungstraditionen erkennen. Sowohl in den USA, als auch in Großbritannien hat der Begriff des Liberalismus seit Ende des 19. Jahrhunderts eine massive Umdeutung im Sinne einer "Sozialdemokratisierung des Liberalismusbegriffes" (Biebricher 2018: 11) erfahren. Werden beispielsweise demokratische Kandidat/innen in den USA als liberal kritisiert, so wird ihnen Sozialdemokratismus vorgeworfen, was einen bemerkenswerten Kontrast zur kontinentaleuropäischen Verwendung des Liberalismusbegriffes ergibt, wo „(Wirtschafts) 
Liberalismus" eher als Vorwurf des mangelnden Sozialbewusstseins an sozialdemokratische Parteien gerichtet wird (vgl. Butterwegge $2018 \mathrm{zu}$ „Hartz VI“).

In Anbetracht dieser komplexen Bedeutungskonstellationen von "Neoliberalismus" und insbesondere dessen Verwendung als politischen Kampfbegriff - zumindest im deutschsprachigen Gebrauch - lässt sich die Frage aufwerfen, ob es nicht angemessener oder fruchtbarer wäre, begriffliche Alternativen wie „Neoklassik“ zu verwenden, um eine sozioökonomische Gegenwart und deren Darstellung in Unterrichtsmaterialien zu analysieren.

Auf den Begriff „Neoklassik“ wird beispielsweise in kritischen Lehrbüchern der VWL zurückgegriffen, die im gewissen Kontrast zu einseitigen ${ }^{1}$ Standardlehrbüchern (Mankiw \& Taylor 2012; Samuelson $\&$ Nordhaus 2010, 2017) einen multiparadigmatischen Zugang verfolgen und somit der Neoklassik andere ökonomische Paradigmen gegenüberstellen (vgl. Heine \& Herr 2012; Jäger \& Springler 2012). Die genannten mehrperspektivischen Lehrbücher setzen also bei ökonomischen Argumentationen und Erklärungsansätzen an und arbeiten durch Gegenüberstellungen unterschiedlicher ökonomischer Denktraditionen implizite politische Dimensionen heraus. Der Begriff "Neoliberalismus" wird in diesen Darstellungen tendenziell ausgespart und wenn er doch Verwendung findet, dann wird "die neoliberale Theorie" als Strang der Neoklassik diskutiert, der sich aus dieser entwickeln konnte (vlg. Jäger \& Springler 2012: 21).

Demgegenüber erfährt der Begriff „Neoliberalismus" in der deutschsprachigen Debatte der kritischen Sozialwissenschaft, wie sie sich etwa in der Zeitschrift PROKLA darstellt, den Vorzug gegenüber dem Begriff der „Neoklassik“. Hier wird beispielsweise diskutiert, ob der Neoliberalismus noch als „hegemonial“ zu fassen ist, oder gar als ,autoritär“ zu bestimmen sei (vgl. Konecny 2012; Oberndorfer 2012). Mit dem Begriff „Neoliberalismus“ soll in diesen Diskussionen also eher auf eine politische Theorie und auf eine Praxis verwiesen werden, die wiederum sehr mit ökonomischer Theoriebildung verwoben ist. Bei den vorhin erwähnten kritischen VWL-Lehrbüchern wird hingegen mit dem Begriff der „Neoklassik“ auf ökonomische Denktraditionen verwiesen, die politische Dimensionen implizieren. Dieser Unterscheidung wird in Abschnitt 2.1 „Politische Dimensionen einer Theoriegeschichte: Neoklassik im Neoliberalismus und Neoliberalismus in der Neoklassik" noch näher nachzugehen sein.

\footnotetext{
Eine Kritik der Standardlehrbücher stellt beispielsweise Silja Graupe (2017) bereit, wobei Sie dem Vorwurf der Indoktrination nachgeht und „unbewusst bleibende Beeinflussung " herausarbeitet.
}

Neben diesen beiden sich weitgehend überschneidenden, aber auch konkurrierenden Begriffen „Neoliberalismus" und "Neoklassik“ kann noch auf ein ganzes Bündel alternativer Begriffe verwiesen werden, um sozioökonomische Verhältnisse und deren Darstellung in Unterrichtsmaterialien zu bestimmen. Auf einem höheren Abstraktionsniveau würden sich etwa „Marktwirtschaft“ oder „Kapitalismus" anbieten. Einmal davon abgesehen, dass diese Begriffe ebenfalls jeweils eine starke Wertung implizieren - zum einen wird der Markt als vermeintlich wertfreie regulatorische Instanz inszeniert und zum anderen wird betont, dass die gewinnbringende Verwertung des Kapitals maßgeblicher ist als menschliche Bedarfe und Bedürfnisse - so zielen diese Begriffe auf sehr allgemeine Bestimmungsmerkmale des Wirtschaftssystems und lassen ein gewisses zeitdiagnostisches Potential vermissen. Es müssen also Fragen nach der spezifischen Ausformung der Marktwirtschaft beziehungsweise des Kapitalismus offenbleiben. Hierfür würden sich neben „Neoliberalismus“ Begriffe, wie „Spätkapitalismus“, „Postfordismus“, „advanced liberalism“ (fortgeschrittener Liberalismus), oder "Toyotaismus" (Ōno 1988) anbieten.

Während „Spätkapitalismus“ zwar betont, dass es sich um eine historisch spezifische Spielart des Kapitalismus handelt - was der Begriffe "Neoliberalismus" vermissen lässt - so läuft das Präfix „spät“, welches auf ein baldiges Ende des Kapitalismus verweist, Gefahr einen Geschichtsdeterminismus zu transportieren. Die weiteren begrifflichen Alternativen weisen ebenfalls entsprechende Schwächen auf. Sie verweisen entweder auf einen Bruch mit Vergangenem, ohne das Neue zu spezifizieren („Postfordismus“, „advanced liberalism") oder sind zu spezifisch. Im Fall von "Toyotaismus“, können zwar Veränderungen im Produktionsprozess zum Ausdruck gebracht werden, aber weitere Dimensionen einer sozioökonomischen Gegenwart - die zwar nicht unabhängig vom Produktionsprozess sind - bleiben zumindest begrifflich verschleiert.

Zusammenfassend lässt sich festhalten, dass der Begriff „Neoliberalismus“ in einem Problemfeld zu verortet ist, wobei zwei Dimensionen dieses Problemfelds erkennbar sind: Zum einen ist der Begriff „Neoliberalismus" Träger von teilweise konkurrierenden Bedeutungen, die von Polemik bis zum akademischen Analysebegriff reichen. Zum anderen lässt sich „Neoliberalismus" auch in einem Netz konkurrierender Begriffe mit teilweise weitgehenden Bedeutungsüberschneidungen erkennen. Im Zuge einer weiterführenden Diskussion des analytischen Potentials des Neoliberalismus-Begriffs scheint es sinnvoll, der bereits angedeuteten Spannung zwischen Neoklassik und Neoliberalismus ideengeschichtlich weiter nachzugehen. 


\section{Politische Dimensionen einer Theoriege- schichte: Neoklassik im Neoliberalismus und Neoliberalismus in der Neoklassik}

In den 1870er Jahren erschienen ungefähr zeitgleich wirkmächtige Arbeiten von Karl Menger, William St. Jevons und Léon Walras (vgl. Heine \& Herr 2012: 11), die weitgehend als „Väter der neoklassischen Theorie“ (vgl. Pierenkemper 2012; Ziegler 2008) bezeichnet werden. Das Präfix „neo“ ist angelehnt an die in England bis dahin vorherrschende Klassische Politische Ökonomie, die beispielsweise den Freihandel betonte (vgl. Konzept des komparativen Vorteils, Ricardo (2004 [1817])). Diese stand nicht nur mit der in Deutschland und Österreich vorherrschenden Historischen Schule in Konkurrenz, die unter anderem in Außenhandelsfragen konträre Positionen bezog ${ }^{2}$. Die Klassische Politische Ökonomie wurde insbesondere auch von Karl Marx kritisiert, der sich der damals vorherrschenden ökonomischen Argumentationsweise bediente und innerhalb dieses Paradigmas eine grundsätzliche Kritik formulierte (Marx 2005 [1867]). Inspiriert von der marxschen Kritik konterkariert beispielsweise Ferdinand Lassalle ${ }^{3}$ mit dem Begriff des „Nachtwächterstaates“ wirkmächtig das wirtschaftspolitische „Laissez-faire“ (Frédéric Bastiat) des klassischen Liberalismus. Der Einfluss solcher Kritik am Liberalismus zeigt sich auch an prominenter Stelle in den späten Werken John Stuart Mills, worin sich ein Wandel vom klassischen Liberalismus hin zu einer sozialdemokratischen Öffnung niederschlägt (vgl. Biebricher 2018: 28).

Unter diesem Druck - sowohl auf die wirtschaftheoretische Klassik, wie auch auf den politischen Liberalismus - kamen also die genannten Arbeiten aus den 1870er Jahren gerade zur rechten Zeit. Das in den 70er Jahren des 19. Jahrhunderts aufkommende neoklassische Paradigma verdrängte mit dem 1890 von Alfred Marshall (2014 [1890]) herausgebrachten „Principles

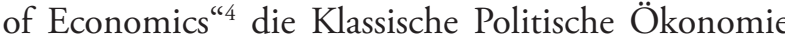
weitgehend und distanzierte sich zwar in vielerlei

\footnotetext{
2 Die Historische Schule konzentrierte sich auf Fragen der „nachholenden Entwicklung" und entwickelte das "Schutzzollargument" gegen die Argumentation des internationalen Freihandels, um damit zu hervorzuheben, dass rückständigere Ökonomien durch staatliche Maßnahmen wie Zölle den Aufbau gewisser Industrien fördern können (vgl. List 2008 [1841]).

3 einer der einflussreichsten Arbeiterführer des 19. Jahrhunderts; einer der Gründerväter der SPD; Seine Vorstellung des Sozialismus war u.a. preußisch-nationalstaatlich geprägt und unterschied sich somit insbesondere zu einer internationalistischen Ausprägung, wie sie Marx und Engels vertraten.

4 Marshalls „Principles of Economics“ bildet bis heute den Kern neoklassischerTheorie(vgl.Jäger\&Springler2012:37) undwirdauch in aktuellen Volkswirtschaftslehrbüchern (vgl. Mankiw \& Taylor 2012) in ähnlicher Form dargestellt (kritisch hierzu: Graupe 2017).
}

Hinsicht von dieser ${ }^{5}$, doch bestätigte und radikalisierte das neue Paradigma den klassischen Zugang gleichzeitig. So verschwand der Bezug auf die Kategorie der Klassen und wurde durch Kategorien wie Haushalte bzw. Individuen und Unternehmen ersetzt. Gesellschaftstheoretische Zugänge wurden von einem „methodologischen Individualismus" und dem Konzept des „Homo oeconomicus“ abgelöst. Außerdem wurde Ökonomie von Politik getrennt und als eigenständige Sphäre, im Sinne einer „reinen Ökonomie“ betrachtet (Screpanti \& Zamagni 2005: 165 ff.). In dieser Zeit setzt auch die Institutionalisierung der Ökonomie an den Universitäten verstärkt ein. Neu geschaffene Lehrstühle wurden bevorzugt von Vertreterinnen und Vertretern der Neoklassik besetzt, wodurch die Klassische Politische Ökonomie und die Historische Schule auch im akademischen Bereich zunehmend marginalisiert wurden (vgl. Jäger \& Springler 2012: 19).

Während Ende des 19. Jahrhunderts zwar ein Methodenstreit ${ }^{6}$ zwischen Historischer Schule und der Neoklassik geführt wurde, so kam es doch bis zu den 1920/30er Jahre zu einer breiten Durchsetzung und Ausdifferenzierung des Paradigmas in Theorie und Praxis in kapitalistischen Regionen (vgl. Jäger \& Springler 2012: 19-20). Ab den 1930er Jahren geriet die Neoklassik nach der Wirtschaftskrise, unter dem aufkommenden keynesianischen Paradigma wieder unter erheblichen Druck. Dieses nach John Maynard Keynes benannte Paradigma geht - im Kontrast zum neoklassischen Paradigma - davon aus, dass die kapitalistische Produktionsweise zu Instabilität tendiere und Staatsinterventionen zur Stabilisierung unerlässlich sind.

Die Reaktion auf den sich verbreitenden Keynesianismus ließ nicht lange auf sich warten. Auf der Ebene der ökonomischen Theoriebildung wurde bereits kurz nach der Erscheinung von Keynes' (1964 [1937]) Werk „The General Theory of Employment, Interest, and Money" versucht diesen Ansatz zu vereinnahmen. So hat John Hicks (1937) im gleichen Jahr den Aufsatz „Mr. Keynes and the „Classics": A Suggested Interpretation" herausgebracht und dabei versucht Keynes" Theorie in das „Investment-Saving/Liquidity-MoneyModell“" (IS-LM-Model) zu integrieren. In diesem Modell wird angenommen, dass auf lange Sicht eine Volkswirtschaft sich einem natürlichen Gleichgewicht annähert - was in einem krassen Widerspruch zu Keynes' Annahme steht - und dadurch nachfragefördernde wirtschaftspolitische Eingriffe von Seiten des Staates obsolet wären (vgl. Jäger \& Springler 2012: 21).

So baut Marshall beispielsweise auf den Arbeiten zur Wertbzw. Preistheorie von Karl Menger, William St. Jevons und Léon Walras auf, die einen grundlegenden Bruch mit der in der Klassik vorherrschenden Arbeitswerttheorie darstellen (vgl. Heine \& Herr, 2012, S. 11-13).

6 Näheres siehe hierfür z. B. Söllner (2012: 219-224) 
Entscheidender als die Aneignung weiter Teile der keynesianischen Theorie scheint jedoch die Gründung der Mont Pèrlerin Society in den 1940er Jahren, was Biebricher (2018) als „zweite Geburt des Neoliberalismus" bezeichnet. Diese Gründung kann als Konstituierung eines Forums zur strategischen Vernetzung und zur Durchsetzung entsprechender Sichtweisen und Wirtschaftspolitiken verstanden werden (vgl. Plehwe \& Walpen 1999; Walpen 2004). In dieser Gründungsphase verwenden wichtige Mitglieder, wie Walter Eucken, Wilhelm Röpke und Friedrich August von Hayek, „neoliberal“ offensiv als Selbstbeschreibung. Darüber hinaus konstituiert sich der Neoliberalismus spätestens mit der Gründung dieses Netzwerks auch als politisches Projekt.

Von diesem ideengeschichtlichen Abriss soll für den weiteren Gang der Argumentation neben den genannten Aspekten neoklassischer und neoliberaler Theorie auch die Verwendung von "neoliberal“ als Selbstbeschreibung festgehalten werden. Darüber hinaus sollte gezeigt werden, dass die ökonomische Theoriebildung stark mit der Bildung politischer Theorie verwoben ist. Ideengeschichtlich lässt sich auf der Ebene der ökonomischen Theoriebildung wohl die Hervorbringung der Neoklassik kaum ohne die Krise des klassischen Liberalismus auf der Ebene der politischen Theoriebildung erklären und umgekehrt die Hervorbringung des Neoliberalismus wohl kaum ohne vorangegangener ökonomischen Theoriebildung seitens der Neoklassik. In dieser historischen Rekonstruktion der Verquickung aus ökonomischer und politischer Theoriebildung wurde auch versucht, die Ebenen der jeweiligen Theoriebildung mit politischer Praxis (neoklassische Lehrstühle, Mont Pèrlerin Society) zu verweben. Um diese Verflechtungen aus ökonomischer und politischer Theoriebildung mit politischer Praxis begrifflich zu fassen, bietet es sich an, von einem „neoliberalen Projekt“ zu sprechen.

Lässt man sich nun auf „neoliberales Projekt" als analytischen Begriff ein, so ist darauf hinzuweisen, dass hierbei ein heterogenes Phänomen in den Blick gerät. Sowohl auf der Ebene der Theoriebildung ${ }^{7}$ als auch auf der Ebene der gesellschaftlichen Transformation $^{8}$ lässt sich ein breites Spektrum an teils kontroversen Positionen und unterschiedlichen Entwicklungen erkennen. Dennoch erachten wir es nicht als sinnvoll „Neoliberalismen“ im Plural zu untersuchen, da

\footnotetext{
So unterscheiden sich beispielsweise der Ordoliberalismus nach Eucken vom Monetaristischen Neoliberalismus nach Friedman teilweise erheblich.

8 Es scheint auch sinnvoll, zwischen Transformationsprozessen, wie sie in Chile in den 1970er Jahren von Pinochets Militärdiktatur durchgesetzt wurden, und ähnlichen Maßnahmen, die etwa im Neuseeland der 1980er Jahre von einer liberal-demokratischen Regierung verabschiedet wurden, zu unterscheiden.
}

das zentrale und verbindende Ziel des „neoliberalen Projekts" einheitlich die Durchsetzung einer Marktgesellschaft ist (vgl. Biebricher 2018; Crouch 2011; Harvey 2012). Während die gemeinsame Vorstellung einer Marktgesellschaft auf ein uneingeschränktes Primat der Ökonomie gegenüber der Politik hinausläuft, oder auch private zwischenmenschliche Beziehungen letztlich als Tauschverhältnisse entwertet ${ }^{10}$, zeigen sich die angedeuteten Varianzen im „neoliberalen Projekt“ hinsichtlich der konkreten Hervorbringung und der Praktiken der Durchsetzung der Marktprinzipien. Auf zwei dieser Varianten wird nun näher eingegangen.

In der Denktradition des deutschen Neoliberalismus (auch Ordoliberalismus) wird der Markt als staatliche Veranstaltung (Miksch 1947) gedacht. Hierbei wird paradoxerweise nach einem starken Staat verlangt, dessen Wirken jedoch auf die Konstituierung und Sicherung von Märkten beschränkt ist: „Die wirtschaftspolitische Tätigkeit des Staates sollte auf die Gestaltung der Ordnungsformen der Wirtschaft gerichtet sein, nicht auf die Lenkung des Wirtschaftsprozesses" (Eucken 2004). Dies soll etwa durch eine "Wirtschaftsverfassung" (vgl. Böhm 2010) gesichert werden, um den Spielraum staatlicher Eingriffe zu begrenzen und die übrigen Akteurinnen und Akteure auf ein Handeln im Rahmen des Marktes zu verpflichten. Der eben zitierte Franz Böhm spitzt dies auf die zynische Behauptung: „Freiheit nur im Rahmen der Ordnung" (Böhm 2010: 101) zu. Das Spannende an dieser ordoliberalen Idee des Marktes als staatliche Veranstaltung ist diese Paradoxie, dass einerseits Freiheit in den Marktbeziehungen vorausgesetzt wird, aber andererseits wohl wenig Vertrauen bestand, dass diese auf Konkurrenz basierenden Marktbeziehungen tatsächlich akzeptiert und freiwillig eingehalten würden - daher auch das Verlangen nach einem starken Staat, als Veranstalter dieser unfreiwilligen „Freiheit“.

Während der deutsche Neoliberalismus den Kapitalismus und den Markt explizit ordnungspolitisch durchsetzen bzw. sichern wollte, versucht Hayeks evolutionärer Erklärungsansatz den Markt als spontane Ordnung argumentativ zu entpolitisieren. Hayek erachtet den Konkurrenzkampf selbst als Ordnungsprinzip der Gesellschaft. Während er sich vom Laissez-faire-Gedanken des Wirtschaftsliberalismus vom 19. Jahrhundert abgrenzt und ebenfalls einen bestimmten Ordnungsrahmen für notwendig hält, ist er jedoch skeptischer hinsichtlich einer staatlich betriebenen Wettbewerbspolitik. Um dies zu argumentieren rahmt er den Wettbewerb als ein „Entdeckungs-

\footnotetext{
9 Kritisch hierzu Polanyi (1944), Hacker (2018) und Marsili \& Milanese (2019)

10 Gary S. Becker (1993) beschreibt diese Sichtweise etwa in seinem Werk „Der ökonomische Ansatz zur Erklärung menschlichen Verhaltens".
} 
verfahren" (Hayek 1994), als einen Prozess der nicht im Voraus bestimmbar ist und dessen Ergebnisse offen sein müssen, um entsprechende Dynamiken entfalten zu können. Es geht Hayek also weniger darum den Wettbewerb zu organisieren als ihn zu arrangieren. Hayek erachtet dabei eine zielorientierte Gestaltung von Wirtschaft und Gesellschaft weder als möglich noch als wünschenswert. Er kritisiert eine zielorientierte Gestaltung („konstruierte Ordnung“) mit der Begrenztheit des Wissens: Der Mensch wäre schlicht nicht in der Lage eine komplexe soziale Ordnung zu entwerfen. Was Wissenschaft und Politik nicht können, vermag dann der Wettbewerb durch eine hervorgebrachte „spontane Ordnung“. Aus dieser „Katallaxie“, wie Hayek seine Vorstellung der spontanen Ordnung auch nennt, könnten sich individuelle Handlungen frei von Zielvorgaben entfalten. Aus der Summe dieser Handlungen würden Regelmäßigkeiten erkennbar werden, die allmählich zu anerkannten Regeln führten. Das Besondere an diesem Gedankengang stellt für Hayek das Hervorgehen einer Ordnung dar, die frei von hierarchisierten Zielvereinbarungen ihrer Mitglieder ist (vgl. Niechoj \& Wolf, 2000). Mit diesem Konzept der spontanen Ordnung ist es Hayek gelungen, die teilweise religiös geladene Legitimation des Marktes (unsichtbare Hand) der Klassik folgenreich zu rehabilitieren. Hayek nutzt in seiner Argumentation auch eine berechtigte Kritik an der Unterdrückung von Individuen in totalitären Gesellschaftsexperimenten des 20. Jahrhunderts, wobei er jedoch mit seinem Gedankengang selbst in einer totalitären Ordnung landet: Seine spontane Ordnung des Marktes schließt Ziel-Mittelansätze hinsichtlich gesellschaftlicher Gestaltung aus - mit weitreichenden Folgen: „Das verfolgen positiver, kollektiver Ziele - etwa Vollbeschäftigung oder soziale Gerechtigkeit - gilt als gegen den Zivilisationsprozess gerichteter Konstruktivismus. Damit beschränkt sich das menschliche Dasein auf die Möglichkeit zur individuellen Anpassung an die spontane Ordnung, der sich die Menschen unterzuordnen haben"(Ptak 2017: 44).

Um diesen Konturen des neoliberalen Projekts weiter nachzugehen, wird im Folgenden eine strukturgeschichtliche Perspektive eingenommen, wobei gefragt wird, ob bzw. inwiefern das neoliberale Projekt nach dessen „zweiter Geburt“ in den 1940er Jahren zur Blüte gefunden hat.

\section{Gesellschaftliche Neoliberalisierungspro- zesse (Fokus: „Europäische Union“)}

Exemplarisch für einen neoliberalen Transformationsprozess, also eine zunehmende Durchsetzung einer Marktgesellschaft, wird der Fokus der fol- genden strukturgeschichtlichen Perspektive auf die Europäische Union gerichtet. Um eine Analyse des Einigungsprozesses der EU jenseits eines methodologischen Nationalismus zu ermöglichen und somit die im Rechtspopulismus verbreitete dichotome Gegenüberstellung der jeweiligen Nationalstaaten mit der $\mathrm{EU}$ vorzubeugen, die den Eindruck erweckt, dass es die „Europäische Union“ im Nationalstaat nicht gebe, verwenden wir das Konzept des ,europäischen Staatsapparate-Ensembles" (kurz: ESE) (Buckel, Georgi, Kannankulam \& Wissel 2014). Aus dieser Perspektive wird der Wandel der EU aus einem Zusammenspiel unterschiedlicher Maßstabsebenen erklärt.

Dieser Wandel der Integrationsweise, also des diskontinuierlichen und unabgeschlossenen Einigungsprozesses, des ESE wird beispielsweise von Ziltener (1999) auf eindrucksvolle Weise analysiert. In seiner Untersuchung des Strukturwandels des ESE arbeitet Ziltener zwei idealtypische Formen von Staatlichkeit (im ESE) heraus. Für die Phase von der Nachkriegszeit bis in die 1970er Jahre spricht er dabei von einer „keynesianisch-korporatistischen Staatlichkeit“" (Ziltener 1999: 50), was auf die keynesianisch geprägte Wirtschaftspolitik mit wohlfahrtsstaatlichen Zielen wie die Schaffung von Vollbeschäftigung, die Angleichung regionaler Lebensstandards oder die Reduktion des Inflationsdrucks - im Kontext eines relativ deckungsgleichen Akkumulations- und der Regulationsraums - verweist. Mit „korporatistisch“ betont Ziltener ein sozialpartnerschaftlich vermitteltes Verhältnis zwischen Arbeit und Kapital mit relativ starken Gewerkschaften (vlg. Ziltener 1999: 50-53).

Im Verlauf der Krisen der 70er und 80er Jahre und damit verbundene ökonomische und gesellschaftliche Veränderungen geriet der keynesianisch-korporatistische Wohlfahrtsstaat in mehrfacher Hinsicht unter Druck: Durch die Internationalisierung der Produktionskreisläufe und Zirkulationsflüsse zerbrach die relative Einheit zwischen Akkumulationsraum und Regulationsraum, was oft als Ende der „fordistischen Produktionsweise" (Lipietz 1997) diskutiert wird. Die zentralen politischen Zielsetzungen (Vollbeschäftigung, Wirtschaftswachstum, oder Preisstabilität) konnten nur mehr selektiv erreicht werden. Hinzu kam, dass sich die verschiedenen Krisenelemente als Finanzkrise des Nationalstaates niederschlugen. Von 1973-1979 stand bei den Versuchen der Krisenbewältigung noch die Stimulierung der inneren Nachfrage nach keynesianischem Modell im Vordergrund. Doch die Regulationskapazitäten der Nationalstaaten erfuhren in Anbetracht eines zunehmend verselbstständigten Weltmarktes ihre Grenzen. Nicht zuletzt auf Grund einer politischen Wende in Großbritannien („thatcherism“) reagierten die westeuropäischen Länder, die ehemals noch mit weitgehend ähnlichen 
Regulationsweisen ausgestattet waren, vorerst mit divergierenden Antikrisenstrategien (vgl. Kohler-Koch 1992; Scharpf 1987; Streeck \& Schmttter 1991).

Ziltener versucht die Entwicklungen deren Ausgangspunkt hier skizziert wurde und zur Herausbildung einer neuen Staatlichkeit führte, mit „Schumpeterianische Wettbewerbsstaatlichkeit" begrifflich zu fassen (Ziltener 1999: 50). Der so beschreibende Staatstyp wird mit dem Nationalökonomen und Politiker Schumpeter verknüpft, der als Theoretiker technologischer und wirtschaftlicher Innovationsprozesse bekannt wurde. Damit ist auch schon ein zentrales Element der sich neu herausbildenden Form von Staatlichkeit benannt - der zunehmende Ausbau von staatlicher Tätigkeit im Bereich von Forschung, Entwicklung und Infrastruktur. Die Herausbildung dieser neuen Form von Staatlichkeit geschieht in einer Zeit einer zunehmend deregulierten interdependenten Weltwirtschaft und einem damit verbundenen Wettbewerbsdruck auf Unternehmen und Standorte.

Dieser strukturelle Wandel hin zum „Wettbewerbsstaat" (Hirsch 1995) bringt auch eine radikale Veränderung der politischen Zielsetzung der Staatlichkeit mit sich. Abgeleitet von der neuen Ausrichtung hin zur Wettbewerbsfähigkeit der Volkswirtschaft, die durch Interventionen auf der Angebotsseite - beispielsweise durch Förderung der Produkt-, Prozess-, Organisations- und Marktinnovation - erreicht werden soll, hat sich die Sozialpolitik den Bedürfnissen einer Arbeitsmarktflexibilität bzw. den Zwängen des internationalen Wettbewerbs unterzuordnen. Dadurch wird eine sozialökonomisch ausgewogene und demokratische innergesellschaftliche Entwicklung zunehmend erschwert (vgl. Ziltener 1999: 56 f.).

Ziltener arbeitet diese drastische Einschränkung demokratischer Entscheidungsmöglichkeiten auf bemerkenswerte Weise heraus. Er zeigt auf, dass die Schaffung eines einheitlichen Binnenmarktes innerhalb des ESE, gekoppelt an das „Prinzip der Gegenseitigen Anerkennung" nationaler Standards in Verbindung mit dem Subsidiaritätsprinzi $p^{11}$ zu einer Logik der „regulatorischen Konkurrenz" (Ziltener 1999: 195) führt. Durch diese Konstellation wurde eine Asymmetrie zwischen Regulations- (auf nationaler Ebene verbleiben Sozial-, Lohn- und Transferpolitik) und Akkumulationsraum (Binnenmarkt, Geld- und Währungspolitik auf europäischer Maßstabsebene) geschaffen, wodurch die unterschiedlichen regulatorischen Instanzen (Mitgliedsstaaten, Städte) in einen Wettbewerb gezwungen werden (Bieling \& Schulten 2003;

\footnotetext{
11 Gibt vor, dass die Europäische Gemeinschaft nur tätig werden darf, wenn die Maßnahmen der Mitgliedstaaten nicht ausreichen und somit die politischen Ziele besser auf der Gemeinschaftsebene erreicht werden können.
}

Bieling \& Steinhilber 2000). Dies ist eine wesentliche Änderung im Verhältnis zwischen Ökonomie und Politik - was Crouch (2008) als „Postdemokratie“ beschreibt. Giersch (1988) bringt diese Veränderungen durch die "wettbewerbsstaatliche Integrationsweise“ (Ziltener 1999: 195) im ESE folgendermaßen auf den Punkt: „Städte konkurrieren mit Städten, Regionen mit Regionen, Steuersysteme mit Steuersystemen. Ähnliches gilt für die Systeme der sozialen Sicherheit mit ihren Leistungen auf der einen Seite, ihren Zwangsabgaben auf der anderen"(Giersch 1988: 10 f.)

Es ist überaus bemerkenswert wie sehr diese strukturgeschichtliche Rekonstruktion des Wandels der europäischen Integrationsweise mit Hayeks Vision hinsichtlich einer Durchsetzung einer Marktgesellschaft deckt, die er folgendermaßen formuliert:

„Wenn Güter, Menschen und Geld frei über die Grenzen hin beweglich sind, so wird es unmöglich, durch eine einzelstaatliche Maßnahme auf die Preise der verschiedenen Erzeugnisse einzuwirken. [...] Es ist weiters klar, daß es nicht möglich sein würde, daß die Staaten innerhalb des Bundes eine selbständige Währungspolitik verfolgen. Mit einer gemeinsamen Währungseinheit wird die Handlungsfreiheit, die den nationalen Zentralbanken gegeben ist, zumindest so beschränkt sein wie unter einer strengen Goldwährung [...]. Auch im rein finanziellen Bereich wären die Methoden zur Erhöhung der Staatseinkünfte für den Einzelstaat einigermaßen beschränkt. Nicht nur würde es die größere Beweglichkeit zwischen den Staaten notwendig machen, alle Arten von Steuern zu vermeiden, die das Kapital oder die Arbeit anderswohin treiben würden; sondern es gäbe auch beträchtliche Schwierigkeiten mit indirekten Steuern" (Hayek 1952: 320-328).

Diese Hayek'sche Vision lässt nun die wettbewerbsstaatliche Integrationsweise im europäischen Staatsapparate-Ensemble mit ihrer impliziten Logik der regulatorischen Konkurrenz auch ideologisch klar einordnen und als erfolgreiche institutionelle Verankerung des „neoliberalen Projekts" erkennen. Wie in Abschnitt 3 angedeutet, begründet sich der klassische Liberalismus als Bewegung des Bürgertums, die sich in erster Linie gegen den Feudalstaat richtet. Das „neoliberale Projekt" - als ökonomische und politische Theorie, Ideologie, und politische Strategie verstanden - will staatliche Interventionen in Produktionsweise zurückdrängen und „den Markt zum universalen, alle Gesellschaftsbereiche übergreifenden Regulierungsmechanismus erheben " (Butterwegge 2017: 124), was letztlich auch eine demokratische Entscheidungsfindung untergräbt (vgl. Crouch 2008).

Neben den zusätzlichen inhaltlichen Konturen, die der Begriff des neoliberalen Projekts im Zuge dieser Diskussion erhielt, sollen folgende zwei Überlegungen festgehalten werden: Das neoliberale Projekt 
wurde durch diesen strukturgeschichtlichen Abriss als erfolgreiches Projekt erkennbar. In den kritischen Sozialwissenschaften wird dieser Erfolg oft mit Gramscis (1991) Konzept der „Hegemonie“ analysiert, wobei gesellschaftliche Transformationsprozesse nach einem Zusammenwirken von Konsens und Zwang untersucht werden. Auch wenn Konsens und Zwang vorerst als Widersprüche erscheinen, so verweist Gramsci mit diesen begrifflichen Gegenpolen auf die berühmten zwei Seiten einer Medaille: Eine hegemoniale Klasse sei gegenüber verbündeten Klassen führend (Konsens) und gegenüber den gegnerischen Klassen herrschend (Zwang), wobei sie doch immer den Ton angibt. Führend wird eine Klasse(nfraktion) indem sie Kompromisse eingeht, „ihre unmittelbaren ökonomischen Ziele überschreitet und Ziele, Werte und Interessen anderer Gruppen aufgreift, zusammenbindet und auf eine gemeinsame Perspektive verpflichtet" (L. Becker, Candeias, Niggemann \& Steckner 2017: 20). Kannankulam (2008) weist in Anlehnung an den marxistischen Staatstheoretiker Nicos Poulantzas (2002) auf eine Abnahme der Konsensorientierung des neoliberalen Projekts hin und arbeitet autoritäre Tendenzen des neoliberalen Projekts heraus. Einer Ausprägung derartiger autoritärer Tendenzen des neoliberalen Projekts im europäischen Integrationsprozess geht beispielsweise Konecny (2012) nach, indem er die Hervorbringung der European Economic Governance ${ }^{12}$ untersucht.

Die zweite Überlegung, die aus dieser strukturgeschichtlichen Rekonstruktion ableiten lässt, schließt an die Argumentation der autoritären Tendenz des neoliberalen Projekts an. Der Charakter der autoritären Tendenz des neoliberalen Projekts zeigt sich weniger in der Form physischer Gewalt, wirft jedoch trotzdem grundlegende demokratiepolitische Fragen auf. In Anlehnung an Oberndorfer (2012) lässt sich die autoritären Tendenz des neoliberalen Projekts auch mit Foucaults Begriff des „Dispositivs“ fassen. Foucaults Konzept des „Dispositivs“ zielt gewissermaßen auf Vorentscheidungen, innerhalb derer sich soziale Praktiken entfalten können (vgl. Foucault 2008: 119 f.). Das neoliberale Projekt konnte im ESE durch die Implementierung der Logik der regulatorischen Konkurrenz solche Vorentscheidungen setzten. Dies zeigt sich etwa am fragwürdigen Erfolg des Begriffs der „Alternativlosigkeit“ als Unwort des Jahres 2010 (Zeit.de 2011). Die vermeintliche „Alternativlosigkeit" wird oft in Situationen ausgerufen, in denen unbeliebte Maßnahmen politisch durchgesetzt werden sollen (vgl. Zeit.de 2010). In solchen Kontexten wird etwa mit dem "Standortwettbewerb“ argumentiert, also die Gefahr betont, dass Kapital abzufließen drohe,

12 Eine kritische Betrachtung von Governance-Ansätzen folgt im anschließenden Abschnitt. wenn nicht gewisse Maßnahmen (z. B. Senkung von Steuern, Kürzung von Sozialleistungen, etc.) ergriffen würden. Der Erfolg dieses StandortwettbewerbArguments, gewissermaßen die Vorentscheidung im Sinne einer konstruierten Alternativlosigkeit, lässt sich auf diese geschaffene Konstellation der „wettbewerbsstaatlichen Integrationsweise" - auf dieses durch den Erfolg des neoliberalen Projekts hervorgebrachten Dispositiv - zurückzuführen.

\section{Neoliberales Regieren und Regiert-Werden}

Während im letzten Abschnitt das neoliberale Projekt als erfolgreich im Sinne von hegemonial bzw. autoritär dargestellt wurde, und somit eine definitorische Rahmung aus der gegenwärtigen politischen Lage und ihrer Pfadabhängigkeit abgeleitet wurde, so wird in diesem Abschnitt der Blick auf veränderte Regierungspraktiken gerichtet. Hierfür scheint es zielführend, zwei durchaus sehr unterschiedliche Forschungsperspektiven zu diskutieren: die Governance-Forschung und die Gouvernementalität-Studien.

In der Governance-Forschung wird beispielsweise der Frage nachgegangen, wie „Governing without Government" (Rhodes, 1996) ermöglicht werden kann. Diese Frage nimmt vielfältige Prozesse vorweg, die im letzten Abschnitt mit der Dezentrierung von Nationalstaatlichkeit im Mehrebenensystem des ESE bereits angeschnitten wurden. Unter dem strukturellen Druck der regulatorischen Konkurrenz wird unter verschärftem Standortwettbewerb die Frage nach Effizienz und Sparpotentialen auch in der Bürokratie immer lauter. Der „Interventionsstaat" (Fisahn 2012: 365) nach keynesianischer Prägung wird zum kostengünstigeren „Moderatoren-Staat" (Biebricher 2018: 158) umgebaut. Hierfür bedarf es Managementinnovationen, die helfen die öffentliche Hand einem unternehmerischen Ideal anzunähern. Entsprechende Ziele eines solchen New Public Managements sind: „Dezentralisierung, Verflachung von Hierarchien, Qualitätsmanagement, Suche nach Kundennähe (!)“ (Osborne \& Gaebler 1997: 23). Besonders das letztgenannte Ziel, welches Bürger/innen als Kund/innen identifiziert, kann Irritation hervorrufen. Hier jedoch einfach mit ökonomischem Imperialismus zu argumentieren, also mit dem Hinweis auf eine Implementierung ökonomischer Handlungslogiken in nichtökonomische Sphären, wäre wohl zu kurz gegriffen, da ein Bedarf an einem erhöhten Serviceniveau nicht immer von der Hand zu weisen ist. Mit den tieferliegenden Folgen solcher Transformationsprozesse befassen sich die Gouvernementalität-Studien.

Die Gouvernementalitätperspektive geht auf eine Reihe von Vorlesungen von Michel Foucault zurück, 
die in der deutschsprachigen Veröffentlichung mit „Geschichte der Gouvernmentalität" betitelt wurden (Foucault 2017a, 2017b). Hierin arbeitet Foucault die Regierungsmacht als eine Subjetivierungsmacht heraus, wobei es nicht darum geht, einem gegebenen Individuum Befehle zu erweisen, sondern darum das Subjekt aus einem Zusammenspiel aus Selbst- und Fremdführung überhaupt erst hervorzubringen (vgl. Lemke 2010). Dieser Prozess der Subjektwerdung wird in den Gouvernmentalität-Studien oftmals in Anlehnung an Althussers (2016) Denkfigur der Interpellation beschrieben. Diesen Prozess der Anrufung bringt Bröckling (2007) folgendermaßen auf den Punkt: „Subjektivierungsregime konfrontieren den Einzelnen mit spezifischen Erwartungen, die er zurückweisen, zu unterlaufen oder einzulösen versucht, denen er aber niemals voll und ganz genügen kann" (ebd.: 28). Dies funktioniert also nicht über „ReizReaktions-Automatismen, sondern erzeugt einen Sog, der bestimmte Verhaltensweisen wahrscheinlicher machen soll als andere" (ebd.: 38).

Dieser Sog forciert die je individuelle Arbeit am „unternehmerischen Selbst“ (Bröckling 2007), dessen typisches Handlungsmuster aus dem Spannungsverhältnis aus Risikobereitschaft und nüchterner Abwägung hervorgeht: (Bedingte) Freiheit ist für das neoliberale Subjekt grundlegend, was Voraussetzung für die volle Ausschöpfung verwertbarer Produktiv- und Innovationskraft ist. Diese Freiheit läuft aus neoliberaler Perspektive jedoch auch Gefahr in unproduktive Projekte zu fließen, wie den kontemplativen Müßiggang. Solcher nichterwünschter Handlungen entgegnet das neoliberale Projekt mit einer Anrufung in Form einer "Responsibilisierung" (Biebricher 2018: 175), einer Schaffung individueller Verantwortlichkeiten. So wird individuelle Freiheit an individuelle Verantwortung geknüpft, was eine Selbstführung und Selbstsorge bestärken soll.

Die Gouvernementalität-Studien argumentieren dabei jedoch keinesfalls auf einer plumpen moralischen Ebene, um in einem Rundumschlag Eigenverantwortung und Selbstführung zu verteufeln. Sie eröffnen vielmehr den Blick auf eine Verantwortlichkeit, die durch Wettbewerb hergestellt wird. Es wird also herausgearbeitet, dass die Logik der regulatorischen Konkurrenz auch auf individueller Ebene greift, was zu einer bemerkenswerten Dynamisierung gesellschaftlicher Verhältnisse (vgl. Rosa 2005) unter einem permanenten zwang der Effizienzsteigerung führt - oder wie es Bröckling etwas dramatischer formuliert: „Der Wettbewerb unterwirft das unternehmerische Selbst dem Diktat fortwährender Selbstoptimierung, aber keine Anstrengung vermag seine Angst vor dem Scheitern zu bannen“ (Bröckling 2007).

\section{Das „neoliberale Projekt" und der Struk- turzusammenhang von Unterricht}

Während beispielsweise Marx im „Kapital“ (MEW 23-25) nach allgemeinen Bestimmungsmerkmalen, bzw. allgemeinen Logiken der kapitalistischen Produktionsweise fragt, geraten mit dem Begriff des „neoliberalen Projekts" Tendenzen einer historisch spezifischen Ausformung der „Marktwirtschaft“ beziehungsweise des „Kapitalismus“ in den Blick. In diesem Artikel wurde das neoliberale Projekt aus unterschiedlichen Perspektiven diskutiert, wodurch unterschiedliche Bedeutungskonnotationen hervorgebracht werden konnten. So wurde „Neoliberalismus“ auf begrifflicher Ebene in den Fokus genommen. Darüber hinaus wurden „Ideen“, die das neoliberale Projekt prägen, Umgestaltungen materieller Verhältnisse (im europäischen Staatsapparate-Ensemble) sowie die Subjektebene diskutiert, um Konturen des neoliberalen Projekts herauszuarbeiten.

Zusammenfassend lässt sich festhalten, dass das Ziel des neoliberalen Projekts, die Durchsetzung einer Marktgesellschaft, insbesondere durch ein Aufbrechen der relativen Einheit zwischen Akkumulationsraum (Binnenmarkt, Geld- und Währungspolitik auf supranationaler Maßstabsebene) und Regulationsraum (auf nationaler Ebene verbleiben Sozial-, Lohn- und Transferpolitik) vorangetrieben werden konnte. Durch entsprechend tiefgreifende Umgestaltungen wurde eine Logik der „regulatorischen Konkurrenz“ (Ziltener 1999: 195) implementiert, was letztlich durch eine konstruierte Alternativlosigkeit eine demokratische Entscheidungsfindung untergräbt (vgl. Crouch 2008). Die Logik der „regulatorischen Konkurrenz“ bringt auch Tendenzen hervor, die etwa Verantwortlichkeiten gesellschaftlicher Institutionen auf Individuen überträgt, wodurch Effizienz- und Nutzenkalkül in immer neue Lebensbereiche vordringen.

Was lässt sich nun von diese Diskussion des „neoliberalen Projekts" auf den in der Einleitung aufgeworfenen "Strukturzusammenhang von Unterricht“ übertragen, wonach Unterricht in einer Wechselbeziehung zu gesellschaftlichen Verhältnissen steht (Kron et al. 2014: S. 25)?

Um dies näher auszuführen, soll erneut Gramscis (1991) Konzept der „Hegemonie“ aufgegriffen werden. Wie dargestellt: Um Hegemonie zu erringen, sind auch „Werte und Interessen anderer Gruppen [...aufzugreifen, zusammenzubinden], und auf eine gemeinsame Perspektive" zu verpflichten (L. Becker et al. 2017: 20). Candeias (2008) hat diesbezüglich herausgearbeitet, dass etwa „zentrale Forderungen der 68er-, der Frauen-, der Öko- wie der Arbeiterbewegung [... vom neoliberalen Projekt integriert wurden], wodurch aktive Zustimmung organisiert, das kritische 
Potenzial dieser Bewegungen absorbiert und letztlich die Substanz der Bewegungen selbst zersetzt wurde“" (Candeias 2008: 303). Der zentrale Punkt dabei ist, dass entsprechende Interessen aufgegriffen wurden, deren Ziele aber dabei gleichzeitig auf dem Terrain des Neoliberalismus verschoben wurden (vgl. Candeias 2008: 305). In Bezug auf die 68er- und Frauenbewegung argumentiert Candeias, dass die Kritik an "unterdrückenden Fähigkeiten des Wohlfahrtsstaates" (Marcuse 2014: 70) vom neoliberalen Projekt aufgegriffen wurde, jedoch diese staatskritische Stimmung „sozusagen auf den Kopf“ (Candeias 2008: 303) gestellt wurde: Die Kritik an „unterdrückenden Fähigkeiten" (des Wohlfahrtsstaates) konnte zu einer Kritik, die für einen Wohlfahrtsstaats-Abbau diente, verschoben werden. So trafen sich zwar „der reaktionäre Impuls der Neoliberalen und der emanzipative Anspruch der Linken, aber schon unter veränderten Kräfteverhältnissen" (Candeias 2008: 304), sodass letztlich ein hier bereits diskutierter neoliberaler Transformationsprozesse vorangetrieben werden konnte, was eine Umgestaltung von Staatlichkeit (Wettbewerbsstaat) begünstigte und den kritisierten Paternalismus durch Eigenverantwortung ersetzen ließ.

Dieser Zusammenhang aus Konsens (Staatskritik) und Zwang (konkrete politische Umsetzung dieser geteilten Staatskritik in der unbeabsichtigten Hervorbringung von Wettbewerbsstaatlichkeit) lässt sich auch auf eine Analyse von Bildungsinhalten übertragen. So ist etwa für den Bereich der Bildung für Nachhaltige Entwicklung (vgl. Hasse 2006; Danielzik \& Flechtker 2012) denkbar, dass entsprechende Kritik an unnachhaltiger Produktionsweise (als „Bildungsinhalt") im Kontext einer neoliberal geprägten Gegenwart verschoben wird und einen "Bildungsgehalt"13 entfaltet, der das neoliberale Projekt, welches die kritisierte Produktionsweise prägt, letztlich begünstigt. Beispielsweise würde ein Bildungsinhalt, der die Kritik an unnachhaltiger Produktionsweise vorschnell zugunsten von Lösungsvorschlägen aufhebt, wie dies etwa beim Konzept des „politischen Konsumverhaltens" der Fall ist, diesem Muster neoliberaler Instrumentalisierung entsprechen: Das Kritisierte (unnachhaltige Produktionsweise) wird zwar aufgegriffen, dessen Ziel (nachhaltige Produktionsweise) wird jedoch gleichzeitig verschoben, da durch die Hervorbringung von Eigenverantwortung im Sinne des politischen Konsumverhaltens letztlich eine weitere Durchsetzung der Marktgesellschaft, die die kritisierte Produktionsweise erst hervorbringt, vorangetrieben

\footnotetext{
13 Die Begriffe „Bildungsinhalt” und „-gehalt" verwenden wir in Anlehnung an Klafki, jedoch weniger um in Klafkis Tradition des „Exemplarischen Lernens“ (Klafki, 1975) zu argumentieren, sondern um den Strukturzusammenhang von Unterricht in einer neoliberal geprägten Gegenwart herauszuarbeiten.
}

wird. Hinsichtlich des Kritisierten (unnachhaltiger Produktionsweise) wird eben keine Entscheidungsfindung auf gesellschaftlicher Ebene angestrebt, sondern die Problemlösung - vermittelt über Marktprinzipien - auf individuelle Verantwortung abgewälzt (Einkaufszettel als Stimmzettel).

Umgekehrt lassen sich jedoch durch ein Herausarbeiten solcher Hervorbringungen von Eigenverantwortung neoliberale Tendenzen (in Unterrichtsmaterialien) aufdecken. Die Hypothese, die sich hiervon ableiten lässt: Durch ein Aufdecken und Analysieren von Responsibilisierungen, können auch neoliberale Tendenzen in Unterrichtsmaterialien feststellbar werden, die sich aufgrund ihrer Bildungsinhalte (z. B.: Mülltrennung, Gesundheit, etc.) vordergründig keinen ökonomischen Paradigmen zuordnen lassen. Dadurch können auch Unterrichtsmaterialien auf neoliberale Tendenzen untersucht werden, die vordergründig keine ökonomischen Themen transportieren.

Neben einer Analyse neoliberaler Tendenzen von Bildungsinhalten lässt sich dieser Hypothese auch auf die Analyse von Unterrichtspraktiken übertragen. So geht etwa Pongratz (1990) einem Wandel von Disziplinierungstechniken im schulischen Kontext nach. Er arbeitet dabei heraus, dass zu „Beginn des 20. Jahrhunderts ein Übergang von der alten Lern- und Drillschule zu dynamischeren, innengeleiteten Arbeitsformen [...], die darauf hinzielen, möglichst früh Fremd- in Selbstregulierung zu überführen" (Pongratz 1990: 156) einsetzte. Gryl und Naumannn (2016) gehen dieser Hervorbringung von Selbstregulierung in Anlehnung an das „ökonomische Selbst" (Gryl \& Naumann 2016: 19) nach und reflektieren derartige Praktiken aus bildungstheoretischer Perspektive. Mitchell (2018) bezieht den Wandel von Disziplinierungstechniken im schulischen Kontext stärker als Pongratz auf eine neoliberal geprägte Gegenwart und spricht in diesen Zusammenhängen von einer Hervorbringung eines „entrepreneurial child“ (Mitchell 2018: 25).

Das „entrepreneurial child“wird demnach in aktuellen Unterrichtspraktiken zwar in seiner Eigenständigkeit stärker ernstgenommen, jedoch nur insofern dies auch mit einer effizienteren Integration in den vorgegebenen Rahmen der Schule einhergeht. Pongratz bezeichnet dies als eine „Fiktion von Autonomie“ (Pongratz 1990: 156) und vertritt die Auffassung, dass sich hinter derartigen Disziplinierungstechniken die Drillanstalt des 19. Jahrhunderts im neuen Kleide verbirgt, ja gar perfektioniert wurde (vgl. Pongratz 1990: 157).

Ein zentraler Moment eines solchen "Governing without Government" (Abschnitt 5) ist wiederum die Responsibilisierungen. Für weiterführende Analysen hinsichtlich neoliberaler Tendenzen von Unterricht, die auch den "Strukturzusammenhang" (Kron 
et al. 2014: 25) berücksichtigen, also Bildungsinhalte und Unterrichtspraktiken in einem Wirkungszusammenhang mit gesellschaftlichen Verhältnissen analysieren, sollte es sich als hilfreich erweisen, bei derartigen Hervorbringungen von Eigenverantwortung anzusetzen. Die Herausforderung wird darin bestehen, die so herausgearbeiteten Responsibilisierungen, kontextbezogen zu differenzieren: Auch die Förderung eines politischen Subjekts jenseits neoliberaler Verwertungslogiken wird nicht ohne eine Förderung von Eigenverantwortung auskommen. Dabei kann folgende Frage bei dieser graduellen Differenzierung hilfreich sein: Erschöpfen sich die Responsibilisierungen, die wir in einer neoliberal geprägten Gegenwart im schulischen Kontext mitgestalten, in einer gesteigerten Selbstregulierung, die erst durch fehlende gesellschaftliche Verantwortung notwendig wurde, oder sind tatsächlich Freiräume im Sinne von Mündigkeit (Adorno \& Becker 1971) auszumachen?

\section{Acknowledgement}

We acknowledge support by the Open Access Publication Fund of the University of DuisburgEssen.

\section{$7 \quad$ Literatur}

Adorno, T. W. \& H. Becker (1971): Erziehung zur Mündigkeit Vorträge und Gespräche mit Hellmut Becker 1959-1969 (25.). Suhrkamp, Frankfurt.

Althusser, L. (2016): Ideologie und ideologische Staatsapparate (2. Aufl.). VSA-Verl, Hamburg.

Becker, G. S. (1993): Der ökonomische Ansatz zur Erklärung menschlichen Verhaltens (2. Auf.). Mohr, Tübingen.

Becker, L., M. Candeias, J. Niggemann \& A. Steckner (Hrsg.) (2017): Gramsci lesen: Einstiege in die „Gefängnishefte" (Deutsche Originalausgabe, 4. Aufl.). Argument, Hamburg.

Biebricher, T. (2018): Neoliberalismus (3. Aufl.). Junius, Hamburg.

Bieling, H.-J. \& T. Schulten (2003): Competitive Restructuring and Industrial Relations within the European Union. In: Cafruny, A. W. \& M. Ryner (Hrsg.): A ruined fortress? neoliberal hegemony and transformation in Europe. Md: Rowman \& Littlefield Publishers, Lanham.

Bieling, H.-J. \& J. Steinhilber (2000): Die Konfiguration Europas. Dimensionen einer kritischen Integrationstheorie. Westfälisches Dampfboot, Münster.

Birch, K. \& V. Mykhnenko (Hrsg.) (2010): The rise and fall of neoliberalism: The collapse of an economic order? Zed Books, New York.

Böhm, F. (2010): Wettbewerb und Monopolkampf: Eine Untersuchung zur Frage des wirtschaftlichen Kampfrechts und zur Frage der rechtlichen Struktur der geltenden Wirtschaftsordnung (Nachdr. d. 1. Aufl. Heymanns, 1933). Nomos, Baden-Baden.
Bröckling, U. (2007): Das unternehmerische Selbst: Soziologie einer Subjektivierungsform. Suhrkamp, Frankfurt am Main.

Buckel, S., F. Georgi, J. Kannankulam \& J. Wissel (2014): Kämpfe um Migrationspolitik. Theorie, Methode und Analyse kritischer Europaforschung. transcript Verlag.

Butterwegge, C. (2017): Rechtfertigung, Maßnahmen und Folgen einer neoliberalen (Sozial-)Politik. In: Butterwegge, C., B. Lösch \& R. Ptak (Hrsg.): Kritik des Neoliberalismus. S. 123-200. https://doi.org/10.1007/978-3-53120006-4_3

Butterwegge, C. (2018): Hartz IV und die Folgen: Auf dem Weg in eine andere Republik? (3. Aufl.). Beltz Juventa, Weinheim Basel.

Candeias, M. (2008). Von der Dialektik des Neoliberalismus zu den Widersprüchen der Bewegungen. In: Butterwegge, C., B. Lösch, \& R. Ptak (Hrsg.): Neoliberalismus: Analysen und Alternativen. VS Verlag für Sozialwissenschaften, Wiesbaden. S. 301-317.

Crouch, C. (2008): Postdemokratie (Deutsche Erstausgabe; N. Gramm, Übers.). Suhrkamp Verlag, Frankfurtam Main.

Crouch, C. (2011): Das befremdliche Überleben des Neoliberalismus: Postdemokratie II (Deutsche Erstausgabe; F. Jakubzik, Übers.). Suhrkamp Verlag, Berlin.

Eucken, W. (2004): Grundsätze der Wirtschaftspolitik (7. Aufl). Mohr Siebeck, Tübingen.

Fisahn, A. (2012): Den Stier das Tanzen lehren? Europa vor neuen Herausforderungen. In: Prokla 42. S. 357-376.

Foucault, M. (2008): Dispositive der Macht: Über Sexualität, Wissen und Wahrheit. Merve, Berlin.

Foucault, M. (2017a): Geschichte der Gouvernementalität I. Sicherheit, Territorium, Bevölkerung: Vorlesung am Collège de France, 1977-1978 (5. Aufl.). Suhrkamp, Frankfurt am Main.

Foucault,M.(2017b): GeschichtederGouvernementalitätII. DieGeburtder Biopolitik:Vorlesung am CollègedeFrance, 1978-1979 (5. Aufl.). Suhrkamp, Frankfurt am Main.

Giersch, H. (1988): Der EG-Binnenmarkt als Chance und Risiko. Inst. für Weltwirtschaft, Kiel.

Graupe,S.(2017): BeeinflussungundManipulationinderökonomischen Bildung - Hintergründe und Beispiele. S. 125.

Gryl, I. \& J. Naumann (2016): Mündigkeit im Zeitalter des ökonomischen Selbst? Blinde Flecken des Geographielernens bildungstheoretisch durchdacht. In: GWUnterricht 141(1). S. 19-30. https://doi.org/10.1553/ gw-unterricht141s 19

Haarmann, M. P. (2019): Wege zur Emanzipation von der „Ideologie der Gegenwart": Über den Wert historischer Zugänge in der sozioökonomischen Bildung. In: Fridrich, C., R. Hedtke \& G. Tafner (Hrsg.): Historizität und Sozialität in der sozioökonomischen Bildung. S. 159-198. https://doi.org/10.1007/978-3-658-22801-9_8

Hacker, B. (2018): Weniger Markt, mehr Politik: Europa rehabilitieren (1. Aufl.). Dietz, Bonn.

Harvey, D. (2012): Kleine Geschichte des Neoliberalismus (1. Auf.). Rotpunktverlag.

Hayek, F. A. von. (1952): Individualismus und wirtschaftliche Ordnung. Eugen Rentsch, Zürich.

Hayek, F. A. von. (1994): Freiburger Studien: Gesammelte Aufsätze (2. Aufl.). Mohr, Tübingen.

Heine, M. \& H. Herr (2012): Volkswirtschaftslehre: paradigmenorientierte Einführung in die Mikro-und Makroökonomie. Oldenbourg Verlag. 
Hicks, J. R. (1937): Mr. Keynes and the „Classics“; A Suggested Interpretation. In: Econometrica 5(2). S. 147. https://doi.org/10.2307/1907242

Hirsch, J. (1995): Der nationale Wettbewerbsstaat. Id-Verlag, Berlin.

Jäger, J. \& E. Springler (2012): Ökonomie der internationalen Entwicklung: Eine kritische Einführung in die Volkswirtschaftslehre. Mandelbaum.

Kannankulam, J. (2008): Autoritärer Etatismus im Neoliberalismus. Zur Staatstheorie von Nicos Poulantzas. Vsa, Hamburg.

Keynes, J. M. (1964): General Theory of Employment, Interest and Money. Harcourt, Brace, Jovanovich, San Diego.

Klafki, W. (1975): Studien zur Bildungstheorie und Didaktik. Beltz, Weinheim.

Kohler-Koch, B. (Hrsg.) (1992): Staat und Demokratie in Europa: 18. Wissenschaftlicher Kongress der Deutschen Vereinigung für Politische Wissenschaft. Leske + Budrich, Opladen.

Konecny, M. (2012): Die Herausbildung einer neuen Economic Governance als Strategie zur autoritären Krisenbearbeitung in Europa - gesellschaftliche Akteure und ihre Strategien. In: Prokla 42. S. 377-394.

Kron, F. W., E. Jürgens \& J. Standop (2014): Grundwissen Didaktik (6. Aufl.). UTB GmbH, München.

Kuhn, T. (1969): Die Struktur wissenschaftlicher Revolutionen. Surkamp, Frankfurt am Main.

Lemke, T. (2010): Eine Kritik der politischen Vernunft: Foucaults Analyse der modernen Gouvernementalität. Argument Verlag, Berlin.

Lipietz, A. (1997): The post-Fordist world: labour relations, international hierarchy and global ecology. In: Review of International Political Economy 4. S. 1-41. https://doi. org/10.1080/096922997347841

List, F. (2008): Das nationale System der politischen Ökonomie. Nomos, Baden-Baden.

Mankiw, N. G. \& M. P. Taylor (2012): Grundzüge der Volkswirtschaftslehre. Schäffer.

Marcuse, H. (2014): Der eindimensionale Mensch: Studien zur Ideologie der fortgeschrittenen Industriegesellschaft (P.-E. Jansen, Hrsg.). zu Klampen Verlag, Springe.

Marshall, A. (2014): Principles of Economics. http://www. myilibrary.com?id=572183 (o.D.)

Marsili, L. \& N. Milanese (2019): Wir heimatlosen Weltbürger. o.O.

Marx, K. (2005): Das Kapital. Kritik der politischen Ökonomie. MEW 23. Dietz, Berlin.

Miksch, L. (1947): Wettbewerb als Aufgabe. Grundsätze einer Wettbewerbsordnung. Godesberg.

Mitchell, K. (2018): Making workers: Radical geographies of education. Pluto Press, London.

Niechoj, T. \& D. Wolf (2000): Der Mensch als Anpasser. Genese und Evolution von Ordnungen bei Hayek. Marburg.

Nienhaus, L. (2017): Sind Sie neoliberal, Herr Lindner? https://www.zeit.de/2017/26/christian-lindner-fdp-interview/komplettansicht?print (11. März 2019)

Oberndorfer, L. (2012): Die Renaissance des autoritären Liberalismus? Carl Schmitt und der deutsche Neoliberalismus vor dem Hintergrund des Eintritts der "Massen“ in die europäische Politik. In: Prokla 42. S. 413-432.

Ōno, T. (1988): Toyota production system: Beyond largescale production. Productivity Press, Cambridge, Mass.
Osborne, D. \& T. A. Gaebler (1997): Der innovative Staat: Mit Unternehmergeist zur Verwaltung der Zukunft. Gabler, Wiesbaden.

Pierenkemper, T. (2012): Geschichte des modernen ökonomischen Denkens: Große Ökonomen und ihre Ideen. CT: Vandenhoeck \& Ruprecht, Göttingen, Bristol.

Plehwe, D. \& B. Walpen (1999): Wissenschaftliche und wissenschaftspolitische Produktionsweisen im Neoliberalismus. Beiträge der Mont Pèlerin Society und marktradikaler Think Tanks zur Hegemoniegewinnung und -erhaltung. In: Prokla 115. S. 302-236.

Polanyi, K. (1944): The Great Transformation. Politische und ökonomische Ursprünge von Gesellschaften und Wirtschaftssystemen (12. Aufl.). Suhrkamp, Berlin.

Pongratz, L. (1990): Schule als Dispositiv der Macht. Pädagogische Reflexionen im Anschluß an Michel Foucault. In: Vierteljahrsschrift für wiss. Pädagogik 3. S. 289-308.

Poulantzas, N. A. (2002): Staatstheorie. Politischer Überbau, Ideologie, Autoritärer Etatismus. VSA, Hamburg.

Ptak, R. (2017): Grundlagen des Neoliberalismus. In: Butterwegge, C., B. Lösch, \& R. Ptak (Hrsg.): Kritik des Neoliberalismus (3., aktual. Aufl.). Springer VS, Wiesbaden.

Rhodes, R. A. W. (1996): The New Governance: Governing without Government. In: Political Studies 44(4). S. 652-667. https://doi.org/10.1111/j.1467-9248.1996. tb01747.x

Ricardo, D. (2004): The principles of political economy and taxation. Dover Publications, Mineola, N.Y.

Rosa, H. (2005): Beschleunigung: Die Veränderung der Zeitstrukturen in der Moderne. Suhrkamp, Frankfurtam Main.

Samuelson, P. A. \& W. D. Nordhaus (2010): Economics (19. Aufl.). McGraw-Hill, New York.

Samuelson, P. A. \& W. D. Nordhaus (2017): Volkswirtschaftslehre: Das internationale Standardwerk der Makro- und Mikroökonomie: das Standardwerk von Nobelpreisträger Paul A. Samuelson (6. Aufl.). FinanzBuchVerlag, München.

Scharpf, F. W. (1987): Sozialdemokratische Krisenpolitik in Europa. Campus, Frankfurt, New York.

Screpanti, E. \& S. Zamagni (2005): An outline of the history of economic thought (2nd ed. rev. and expanded). http://www.myilibrary.com?id=91434

Söllner, F. (2012): Die Geschichte des ökonomischen Denkens (3. Aufl). Springer Gabler, Berlin.

Streeck, W. \& P. C. Schmitter, P. C. (1991). From National Corporatism to Transnational Pluralism: Organized Interests in the Single European Market. In: Politics \& Society 19(2). S. 133-164. https://doi. org/10.1177/003232929101900202

Walpen, B. (2004): Die offenen Feinde und ihre Gesellschaft: Eine hegemonietheoretische Studie zur Mont Pèlerin Society. VSA-Verlag, Hamburg.

Zeit.de. (2010): Währungskrise. Ohne Alternative? https:// www.zeit.de/2010/51/Euro-Rettung

Zeit.de. (2011): Unwort des Jahres ist „alternativlos“. https://www.zeit.de/gesellschaft/zeitgeschehen/2011-01/ unwort-2010-alternativlos

Ziegler, B. (2008): Geschichte des ökonomischen Denkens: Paradigmenwechsel in der Volkswirtschaftslehre (2. überarb. Aufl.). Oldenbourg, München.

Ziltener, P. (1999): Strukturwandel der europäischen Integration. Westfälisches Dampfboot, Münster. 